\title{
Expression of phosphatase of regenerating liver family genes during embryogenesis: an evolutionary developmental analysis among Drosophila, amphioxus, and zebrafish
}

Ming-Der Lin ${ }^{1,2^{*}}$, Hsun-Tzu Lee ${ }^{5}$, Szu-Chieh Wang ${ }^{1}$, Han-Ru Li ${ }^{4}$, Hsin-Lun Hsien², Kai-Wen Cheng ${ }^{5}$, Yu-Di Chang ${ }^{2}$, Min-Lang Huang ${ }^{3}$, Jr-Kai Yu ${ }^{4^{*}}$ and Yau-Hung Chen ${ }^{5^{*}}$

\begin{abstract}
Background: Phosphatase of regenerating liver (PRL) family is classified as class IVa of protein tyrosine phosphatase (PTP4A) that removes phosphate groups from phosphorylated tyrosine residues on proteins. PRL phosphatases have been implicated in a number of tumorigenesis and metastasis processes and are highly conserved. However, the understanding of PRL expression profiles during embryonic development is very limited.

Results: In this study, we demonstrated and characterized the comprehensive expression pattern of Drosophila PRL, amphioxus $P R L$, and zebrafish PRLs during embryonic development by either whole mount immunostaining or in situ hybridization. Our results indicate that Drosophila PRL is mainly enriched in developing mid-guts and central nervous system (CNS) in embryogenesis. In amphioxus, initially PRL gene is expressed ubiquitously during early embryogenesis, but its expression become restricted to the anterior neural tube in the cerebral vesicle. In zebrafish, PRL-1 and PRL-2 share similar expression patterns, most of which are neuronal lineages. In contrast, the expression of zebrafish PRL-3 is more specific and preferential in muscle.
\end{abstract}

Conclusions: This study, for the first time, elucidated the embryonic expression pattern of Drosophila, amphioxus, and zebrafish PRL genes. The shared PRL expression pattern in the developing CNS among diverse animals suggests that $P R L$ may play conserved roles in these animals for CNS development.

Keywords: Phosphatase of regenerating liver, PTP4A, Embryogenesis, Drosophila, Zebrafish, Amphioxus

\section{Background}

Phosphatase of regenerating liver (PRL) family is classified as class IVa of protein tyrosine phosphatase (PTP4A) that removes phosphate groups from phosphorylated tyrosine residues on proteins. Mammalian PRL family consists of three PRL members. PRL-1 (PTP4A1) was originally identified as an immediate-early growth response gene in the nucleus of regenerating rat liver and mitogen-treated 3T3 mouse fibroblasts [1,2]. PRL-2 (PTP4A2) and PRL-3

\footnotetext{
* Correspondence: mingder@mail.tcu.edu.tw; jkyu@gate.sinica.edu.tw; yauhung@mail.tku.edu.tw

'Department of Molecular Biology and Human Genetics, Tzu-Chi University, No.701, Zhongyang Rd., Sec 3, Hualien 97004, Taiwan

${ }^{2}$ Department of Life Sciences, Tzu-Chi University, No.701, Zhongyang Rd, Sec

3, Hualien 97004, Taiwan

Full list of author information is available at the end of the article
}

(PTP4A3) were subsequently discovered through database searches for sequences homologous to PRL-1 [3].

Overexpression of PRL family members has been implicated in cancer progression. For example, $P R L-3$ up-regulation clearly correlates with colon carcinoma metastases, gastric carcinoma with nodal metastasis, ovarian carcinoma, breast carcinoma, and liver carcinoma cells (reviewed in [4]). Notably, PRL-3 expression level in primary colorectal cancers has prognostic significance in predicting the development of liver and lung metastases [5]. PRL-1 has been found to elevate in renal carcinoma, melanoma, pancreatic cancer cells, and ovarian lymphoma cells [6-8]. In addition to $P R L-1$ and $P R L-3$, $P R L-2$ overexpression is associated with prostate malignancies [9] and breast cancer [10,11]. These observations 
indicate that PRL family proteins play important roles in metastasis and a variety of cancers $[4,12]$.

Although the PRL family members are known to be involved in cancer progression and metastasis, the understanding of normal PRL expression patterns during embryonic development is limited. Most of previous studies were focused on examining PRL expression in adult tissues. For example, rat Prl-1 is mainly expressed in brain, skeletal muscle $[1,13]$, and a number of digestive epithelial tissues [14,15]. Rat Prl-2 mRNA is widely expressed in adult tissues including the anterior pituitary, brain cortex, adrenal gland, kidney, testis, and heart [16]. Rat PRL-3 protein has not yet been examined in adult normal tissues but is found to be expressed in fetal heart [17]. In mouse, Prl-1 mRNA is expressed at all stages examined from E10.5 through E18.5 in a variety of tissues except heart and skeletal muscle [18]. In contrast, mouse Prl-2 mRNA is preferentially expressed in skeletal muscle and Prl-3 mRNA is mainly expressed both in the skeletal muscle and heart [3]. In addition, mouse PRL-3 is also expressed in villus epithelial cells of the small intestine [19]. Similar to mouse Prl-1, human
$P R L-1$ and $P R L-2$ are almost ubiquitously expressed in adult human tissues, except that $P R L-1$ is absent in the brain cortex [20]. In contrast to the ubiquitous expression pattern, human PRL-3 mRNA is most enriched in the heart and skeletal muscle and moderately expressed in the pancreas [21]. These observations indicate that the expressions of PRL members can be varied in a tissue specific manner in mammals.

To our knowledge, no study has yet described and compared the expression patterns of PRLs in Drosophila and zebrafish model animals during embryonic development. Here, we demonstrated and characterized, for the first time, the comprehensive expression pattern of both Drosophila PRL and zebrafish PRLs during embryonic development by either whole mount immunostaining or in situ hybridization. To further understand the evolution of $P R L$ gene in the chordate lineage, we also identify the single $P R L$ orthologue in the basal chordate amphioxus and study its embryonic expression. Our study reveals evolutionary conserved as well as lineage specific PRL expression patterns during embryonic development among Drosophila, amphioxus, and lower vertebrate zebrafish.

Table 1 Summary of PRL/PTP4A family proteins from selected species

\begin{tabular}{|c|c|c|c|c|}
\hline Gene names (species) & Coding region (aa) & $\mathrm{Mw}(\mathrm{kDa})$ & $\mathrm{pl}$ & GenBank accession number \\
\hline \multicolumn{5}{|l|}{ Invertebrate PRL } \\
\hline C. elegans & 190 & 21.16 & 8.97 & NM_076282 \\
\hline Drosophila & 176 & 19.97 & 8.83 & NM_135936 \\
\hline Sea urchin & 179 & 20.44 & 8.98 & XM_001198915 \\
\hline \multicolumn{5}{|l|}{ (S. purpuratus) } \\
\hline Amphioxus & 174 & 19.9 & 9.07 & KC_491215 \\
\hline \multicolumn{5}{|l|}{ (B. floridae) } \\
\hline \multicolumn{5}{|l|}{ Vertebrate PRL } \\
\hline \multicolumn{5}{|l|}{ PRL-1 } \\
\hline Human & 173 & 19.82 & 9.17 & NM_003463 \\
\hline Rat & 173 & 19.82 & 9.17 & NM_031579 \\
\hline Mouse & 173 & 19.82 & 9.17 & NM_011200 \\
\hline Zebrafish & 173 & 20.00 & 9.33 & NM_001007775 \\
\hline \multicolumn{5}{|l|}{ PRL-2 } \\
\hline Human & 167 & 19.13 & 8.67 & NM_080391 \\
\hline Rat & 167 & 19.13 & 8.67 & BC_060549 \\
\hline Mouse & 167 & 19.12 & 8.68 & NM_008974 \\
\hline Zebrafish & 168 & 19.33 & 8.61 & NM_001024098 \\
\hline \multicolumn{5}{|l|}{ PRL-3 } \\
\hline Human & 173 & 19.53 & 9.35 & NM_032611 \\
\hline Rat & 173 & 19.65 & 9.42 & NM_001114405 \\
\hline Mouse & 173 & 19.65 & 9.42 & NM_001166388 \\
\hline Zebrafish & 173 & 19.88 & 9.45 & NM_213181 \\
\hline
\end{tabular}




\section{Results}

Drosophila, amphioxus, and zebrafish PRL phosphatases are highly conserved

PRL phosphatases from selected species and their molecular features are summarized in Table 1. All of these PRL phosphatases possess alkaline isoelectric points (pIs) and are proposed to be positively charged at physiological $\mathrm{pH}$. In vertebrates, PRL family contains three PRL phosphatases whereas there is only one PRL gene in protostomes including C. elegans and Drosophila, and in invertebrate deuterostomes including sea urchin and amphioxus (Table 1). Among vertebrates, PRL homologs including human, mouse, and zebrafish share more than $80 \%$ amino acid sequence identity. For instance, zebrafish PRL-1, PRL-2, and PRL-3 share 92\%, 82\%, and 88\% identities with their human orthologs, respectively. Although a little bit lower, the protostome and invertebrate deuterostome PRLs also share significant amino acid sequence identity and similarity with their vertebrate orthologs. For instance, Drosophila PRL shares 75\% similarity and 58\% identity with human PRL-1 and 74\% similarity and 57\% identity with zebrafish PRL-1.

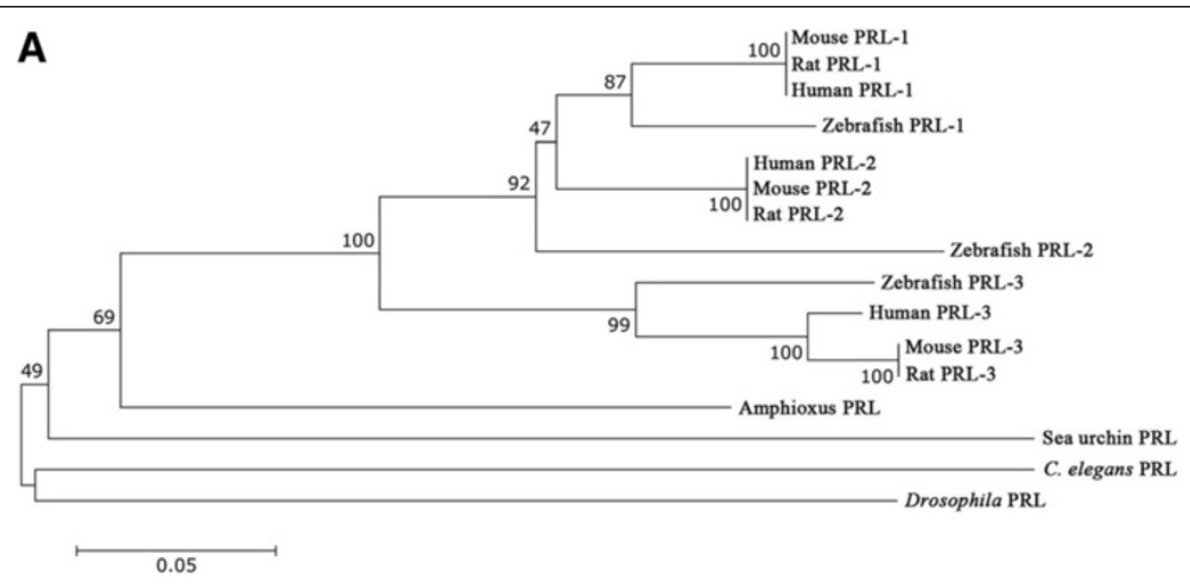

B

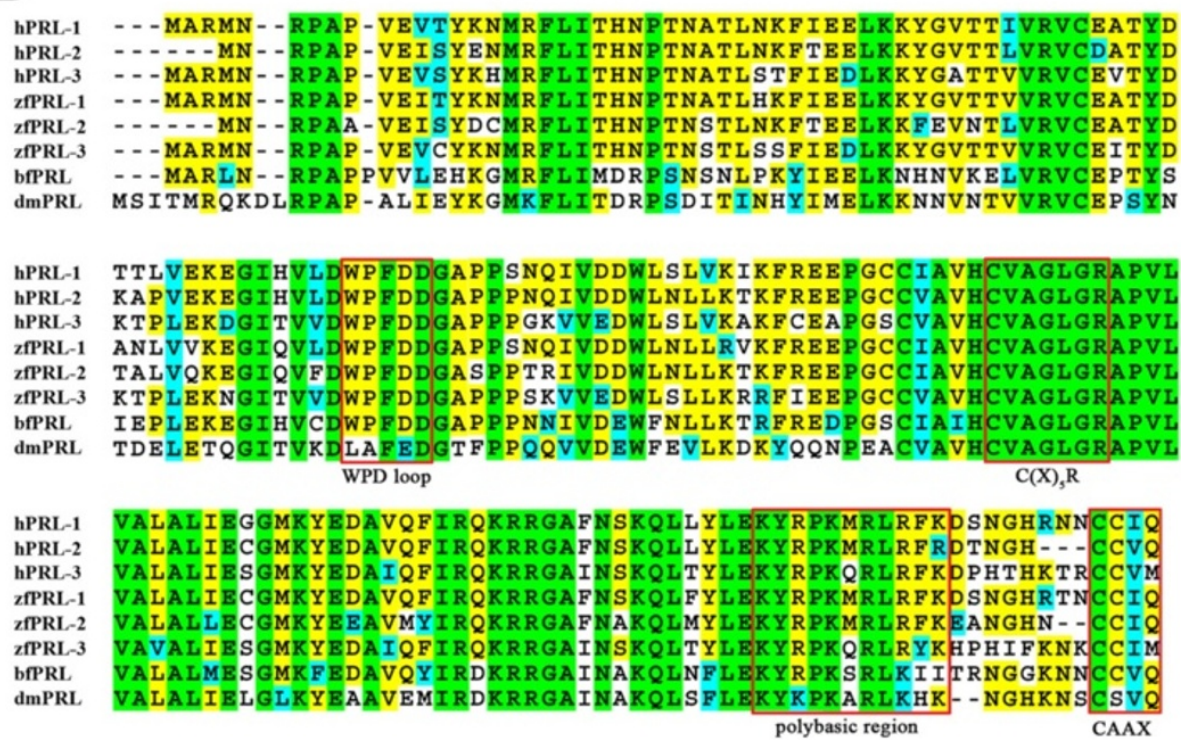

Figure 1 Phylogenetic tree and multiple sequence alignment of PRL protein sequences from human, zebrafish, amphioxus, and Drosophila. (A) Phylogenetic tree of PRL homologue proteins. PRL protein sequences from various species were retrieved from GenBank and corresponding accession numbers are provided in Table 1. The evolutionary history was inferred using the Neighbor-Joining method. The percentage of replicate trees in which the associated taxa clustered together in the bootstrap test (1000 replicates) are shown next to the branches. The tree is drawn to scale, with branch lengths in the same units as those of the evolutionary distances used to infer the phylogenetic tree. The evolutionary distances were computed using the $\mathrm{p}$-distance method and are in the units of the number of amino acid differences per site. Evolutionary analyses were conducted in MEGA5. (B) Multiple sequence alignment of PRL protein sequences from human, zebrafish, amphioxus and Drosophila. Location of conserved catalytic site $C(X)_{5} R$, WPD loop, polybasic region and prenylation motif CAAX box are indicated. Abbreviations: hPRL: homo sapiens PRL, zfPRL: zebrafish Danio rerio PRL, bfPRL: amphioxus Branchiostoma floridae PRL, dmPRL: Drosophila melanogaster PRL. 
To get an idea about the evolutionary relationships of PRL phosphatases among species, we performed phylogenetic analysis. By using neighbor-joining (NJ) method [22] with p-distance model that conducted in MEGA5 [23], vertebrate PRL phosphatases were grouped as a robust clade with $100 \%$ bootstrap support (Figure 1A). This phylogenetic tree demonstrated that vertebrate PRLs originated through gene duplication. Besides, PRL3 phosphatase is classified as a more distinct group from both PRL-1 and PRL-2 phosphatases. Interestingly, the basal chordate Branchiostoma floridae contains only one PRL phosphatase gene in its genome (Table 1), but we identified three cDNA isoforms from our cDNA/EST database. When we aligned these three PRL isoforms to the assembled draft genome sequence, we found they differ in their exon usage for the 5' and 3' UTR region, whereas the exon regions for the protein coding sequence are the same and thus encode an identical protein sequence consisting 174 amino acids (Additional file 1: Figure S1). The single B. floridae PRL sequence branches out at the base of all vertebrate PRLs, suggesting that the duplication events generating PRL-1, PRL-2, and PRL-3 occurred during early vertebrate evolution.

Previous studies indicate that PRL phosphatases contain conserved WPD loop and $\mathrm{C}(\mathrm{X})_{5} \mathrm{R}$ catalytic motif required for its phosphatase enzymatic activity [24,25]. In addition, the C-terminal region consists of a polybasic region together with a C-terminal prenylation motif which are required for its association with plasma membrane and early endosome [19,26,27]. The multiple sequence alignment reveals that Drosophila, amphioxus and zebrafish PRLs also contain these highly conserved signatures (Figure 1B).

\section{Drosophila PRL is expressed in developing mid-gut and CNS in embryogenesis and localized on the plasma membrane}

To analyze the expression pattern of Drosophila PRL $(d m P R L)$ in embryogenesis, we performed whole mount in situ hybridization. At embryonic stage 5, $d m P R L$ is ubiquitously expressed throughout the embryos (Figure 2A). In stage 9 embryos, $d m P R L$ is expressed at developing anterior mid-gut and posterior mid-gut (Figure 2B, arrows). At stage $13, d m P R L$ expression is sustained in developing anterior mid-gut and posterior mid-gut (Figure 2C, arrows). In addition, the expression of $d m P R L$ in ventral nerve cord (VNC) can also be observed at stage $13 \mathrm{em}$ bryos (Figure $2 \mathrm{C}$, arrows). In stage 16 embryos, $d m P R L$ was expressed in a significant amount in procephalon (Figure 2D).

To analyze the expression pattern and subcelluar localization of Drosophila PRL protein, we generated an anti-dmPRL antibody. The full length $d m P R L$ CDS with 176 codons was subcloned into $\mathrm{pET}-32 \mathrm{a}$ vector for
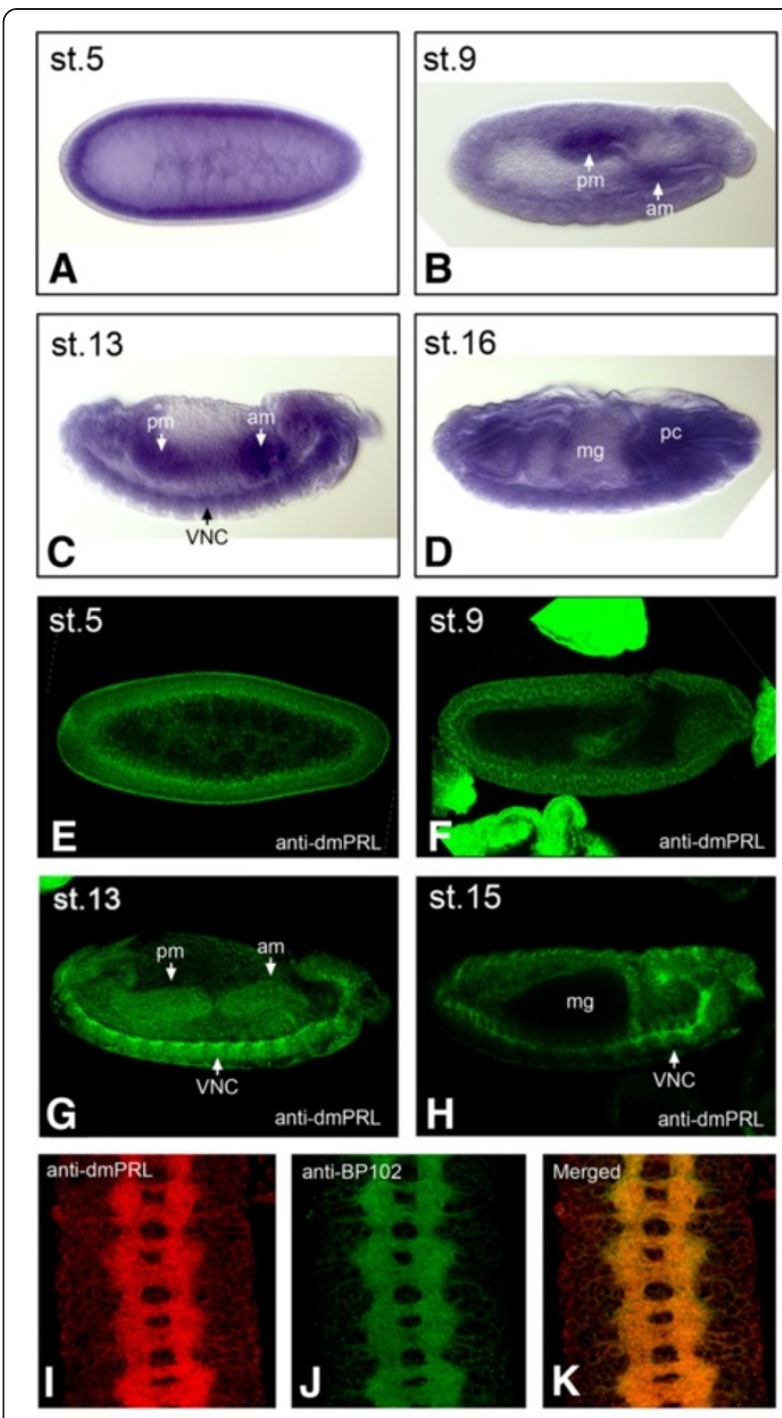

Figure 2 Drosophila PRL is enriched in developing mid-gut and the ventral nerve cord in embryogenesis. (A-D) Whole mount in situ hybridization using antisense probes against Drosophila PRL. (A) dmPRL was ubiquitously expressed in stage 5 embryos. (B) At stage 9, dmPRL was expressed in developing anterior and posterior midguts. (C) At stage 13, dmPRL was expressed in developing anterior and posterior mid-guts together with VNC. (D) At stage 16, the expression of $d m P R L$ was significant in procephalon. (E-H) Whole mount immunostaining of Drosophila PRL during embryogenesis. At stage $5(\mathbf{E})$ and stage $9(\mathbf{F})$, DmPRL was ubiquitously expressed. (G) At stage $13, \mathrm{dmPRL}$ was expressed in developing anterior and posterior mid-guts together with VNC. (H) At stage 15, the expression of dmPRL was enriched in VNC. (I-K) dmPRL (I) and axon marker BP102 (J) were colocalized (K) in VNC of stage 15 embryos. Abbreviations: am, anterior mid-gut; pm, posterior mid-gut; VNC, ventral nerve cord; mg, mid-gut; pc, procephalon.

producing recombinant $\mathrm{dmPRL}$ proteins which were used to generate dmPRL antiserum from rabbits. The dmPRL antiserum preferentially recognized a protein band that represents dmPRL in embryo lysates around $22 \mathrm{kDa}$ on Western blots (Additional file 2: Figure S2, 

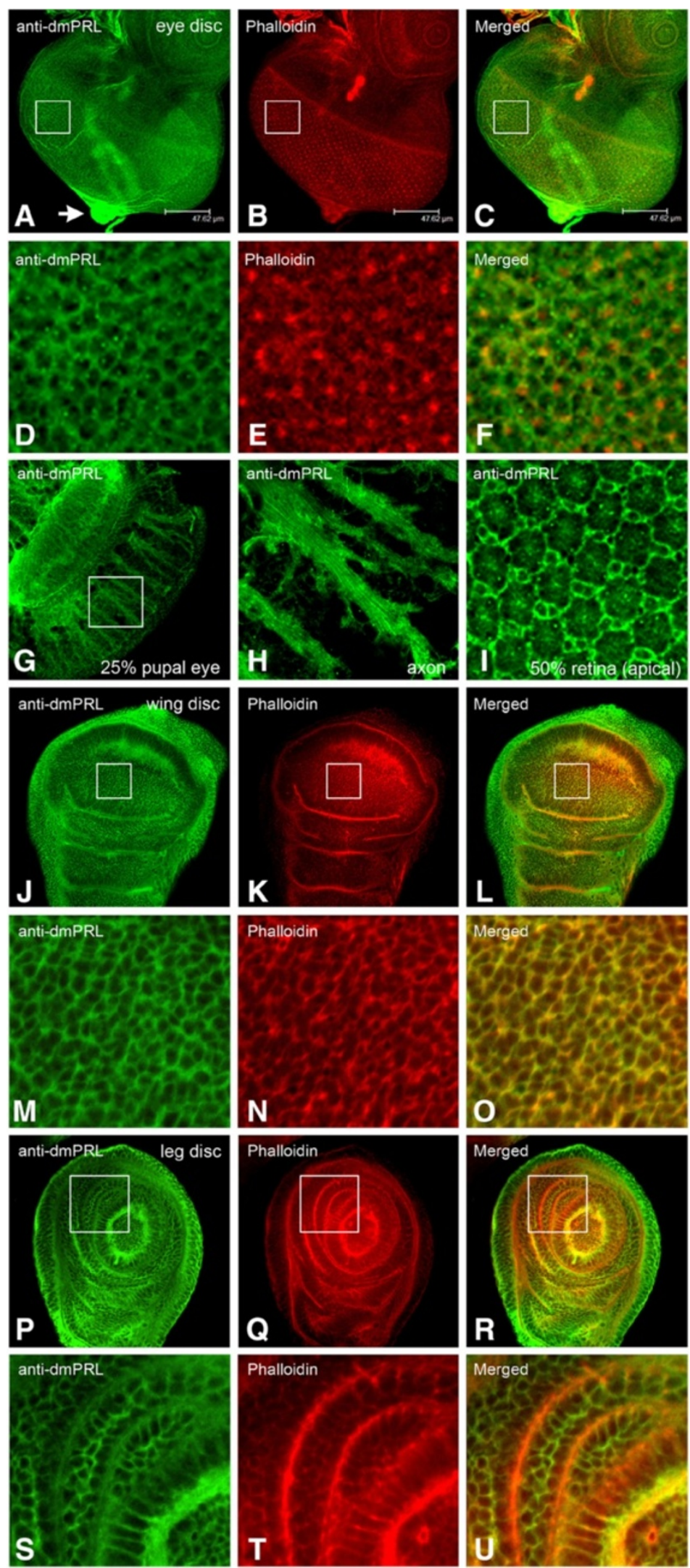

Figure 3 (See legend on next page.) 


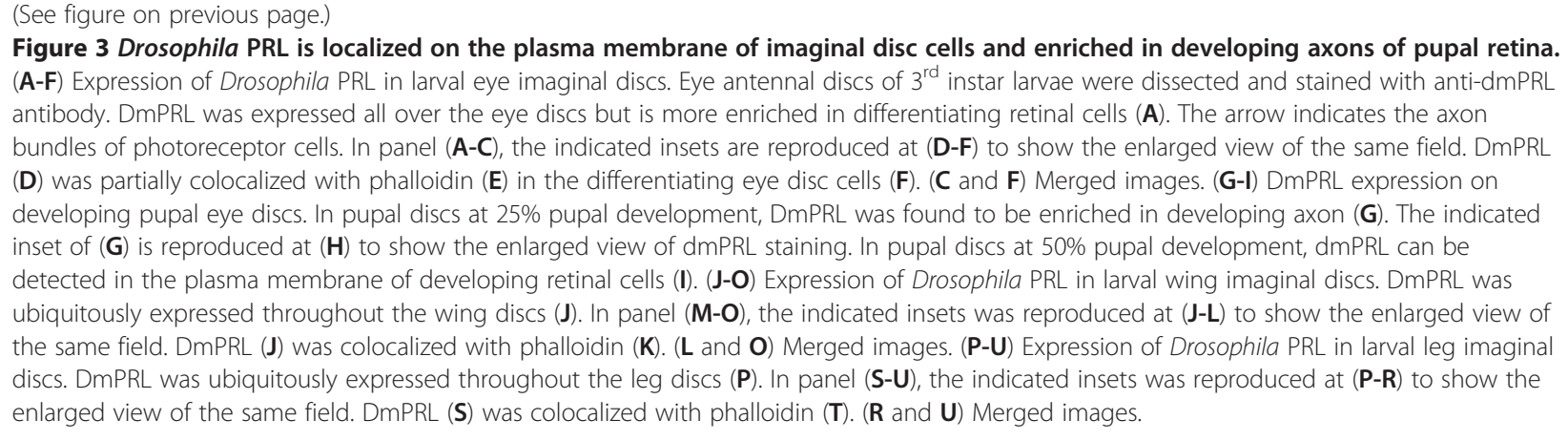

arrowhead). The antibody specificity of dmPRL antiserum was further proved by its recognition of GFP-PRL fusion proteins in ovary lysate (Additional file 2: Figure S2, arrow). To explore the embryonic expression of dmPRL protein, we preformed whole mount immunostaining. At embryonic stage 5 , dmPRL is mainly localized in the apical membrane of blastoderm embryos (Figure 2E). In stage 9 embryos, dmPRL is ubiquitously expressed in the germ band and evenly distributed in the plasma membrane of the cells (Figure 2F). At stage 13, the dmPRL expression is enriched in VNC (Figure 2G). In addition, dmPRL expression can be detected in the developing anterior and posterior mid-guts (Figure 2G, arrows). In stage 15 embryos, dmPRL expression persisted in the VNC (Figure 2H, arrow) but the mid-gut expression of dmPRL had vanished (Figure $2 \mathrm{H}$ ). To confirm the expression of dmPRL in VNC, we dissected VNC of stage 15 embryos and performed double immunostaining with anti-dmPRL and anti-BP102, an axonal marker, antibodies. The dmPRL signal colocalized with BP102 staining perfectly (Figures 2I-K), indicating dmPRL expression is indeed in VNC. In summary, our data suggest that dmPRL begins to enrich in the axon of VNC by embryonic stage 13 .

Previous study indicates that PRL phosphatases contain a C-terminal CAAX prenylation motif which is required for its association with plasma membrane [19]. To further confirm whether dmPRL is localized on the plasma membrane as its human homologs $[19,28]$, we examined the expression of dmPRL on eye imaginal disc. In Drosophila eye disc, dmPRL is more prominent in differentiating disc cells (Figures $3 \mathrm{~A}$ and $\mathrm{D}$ ) and colocalized with phalloidin (Figures $3 \mathrm{~B}$ and $\mathrm{E}$ ) that marks filamentous actin. This result indicates that dmPRL is associated with plasma membrane as its human homologs. In addition, we noticed an enrichment of dmPRL signal in the optic stalk which consisting of photoreceptor axons (Figure 3A, arrow). As the larval eye disc will develop into the future retina, we next examined developing pupal retinas. Similar to larval eye disc, dmPRL signal is highly enriched in the developing axons of pupal eye disc at 25\% pupal development (Figures 3G-H). Further, we also observed that dmPRL can be clearly detected in the plasma membrane of pupal retina at $50 \%$ pupal development (Figure 3I). To examine whether imaginal discs other than eye disc can express dmPRL, we stained wing and leg imaginal discs. In wing discs, dmPRL was ubiquitously expressed (Figures $3 \mathrm{~J}$ and $\mathrm{M}$ ) and colocalized with phalloidin (Figures $3 \mathrm{~K}$ and N). In leg discs, we also observed the ubiquitous expression of dmPRL (Figures 3P and $S$ ) and its colocalized with phalloidin (Figures $3 \mathrm{Q}$ and $\mathrm{T})$. In conclusion, dmPRL is a membrane associated protein and its expression is enriched in developing mid-guts and VNC in embryogenesis.

\section{Zebrafish PRL-1 and PRL-2 are mainly expressed in neuronal cell lineages}

To determine the spatiotemporal expression patterns of PRL-1, PRL-2 and PRL-3 during early development of zebrafish, we performed whole mount in situ hybridization using antisense DIG-labeled riboprobes. Zebrafish PRL-1 transcripts were first detected from 1 4-cell stages to cleavage period (Figure 4A), and extended their expression from the gastrula period to the early segmentation stages (Figures 4B-C). At 24-hpf, the zebrafish $P R L-1$ transcripts were restricted to the head region (Figure 4D). Flat-mount of 24-hpf embryos revealed that PRL-1 signals distributed in the entire brain, including the diencephalon, midbrain, and rhombomeres 1-7 (Figure 4E). By 36- (Figure 4F), 48(Figure 4G) and 72-hpf (Figure 4H), zebrafish $P R L-1$ expressed strongly in brain, eyes as well as in pharyngeal arches (Figures 4F-H). Cryosections of 72-hpf embryo revealed zebrafish $P R L-1$ signals were detected at tectum, iris, retinal pigment epithelium, outer plexiform layer, inner plexiform layer, optic fiber layer (Figure 4I). However, the expression zebrafish $P R L-1$ was relatively weak in somite (Figure 4J) and gut (Figure 4K). These results suggest that the expressions of zebrafish $P R L-1$ transcripts are most abundant at neuronal lineages.

Zebrafish $P R L-2$ transcripts were first observed from 1 4-cell stages to cleavage period, and their expression extended from the gastrula period to the early segmentation 


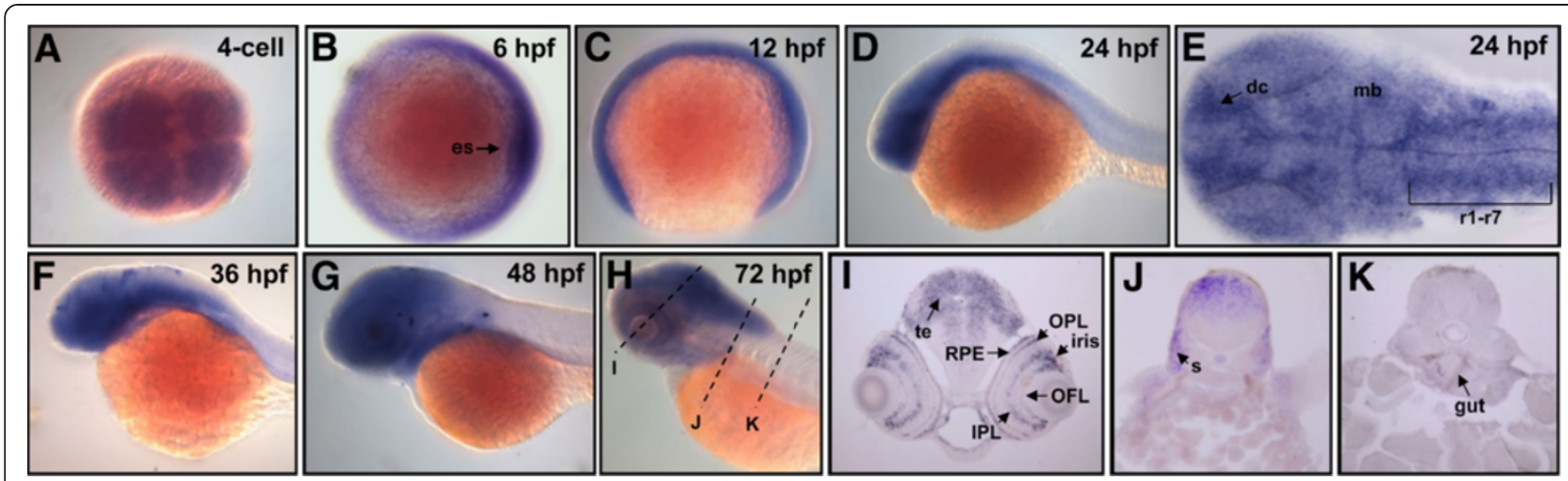

Figure 4 Expression pattern of zebrafish PRL-1 during embryonic development. (A-D, and F-H) Whole mount in situ hybridization using probes against zebrafish PRL-1. Dorsal view of 4-cell stage (A) and 6-hpf (B) embryos. Lateral view of 12-hpf (C), 24-hpf (D), 36-hpf (F), 48-hpf (G), and 72-hpf (H) embryos. (E) Flat mount showed zebrafish PRL-1 signals in the entire brain. (I-K) Cross-sections along the plane indicated by lines showed in (H). Abbreviations: hpf, hours post fertilization; OPL, outer plexiform layer; OFL, optic fiber layer; IPL, inner plexiform layer; RPE, retinal pigment epithelium; dc, diencephalon; mb, midbrain; te, tectum; s, somite.

stages (Figures 5A-C). By 24-hpf, the zebrafish PRL-2 transcripts were detected in head region as well as in trunk (Figures 5D-E). By 36- (Figure 5F), 48- (Figure 5G) and 72-hpf (Figure $5 \mathrm{H}$ ), zebrafish $P R L-2$ expressed strongly in brain, eyes, fin buds as well as in pharyngeal arches (Figures 5F-H). Cryosections of 72-hpf embryo revealed $P R L-2$ signals at outer layer of lens (Figure 5I, arrow), anterior somites (Figure 5J, arrow), and gut (Figure 5K, arrow), but were undetectable at retinal pigment epithelium, inner plexiform layer, optic fiber layer (Figure 5I). On the basis of these observations, we conclude that the neuronal expressions of zebrafish PRL-2 are similar to that of zebrafish $P R L-1$, except that zebrafish $P R L-1$ displays stronger signals in eye regions. In contrast to zebrafish $P R L-1$, zebrafish $P R L-2$ is strongly expressed in gut.
Zebrafish PRL-3 transcripts are expressed in brain, somites, blood island, and head muscles in developing embryos

Zebrafish $P R L-3$ transcripts were first observed at 1-cell stage (Figure 6A). At gastrula stage, $P R L-3$ signals distributed in germ ring and in embryonic shield (es) (Figures 6B-C). By 12-hpf, the zebrafish PRL-3 transcripts were down-regulated suggesting that maternal inherited PRL-3 might be exhausted (Figure 6D). At 24-hpf (Figure 6E), 30-hpf (Figure 6F), and 36-hpf (Figure 6G), the zebrafish $P R L-3$ transcripts were detected at head region. Cryosections of 36-hpf embryo reveals the expression of zebrafish $P R L-3$ transcripts in brain region of mesencephalon and telencephalon (Figure 6H, arrows) and somites (Figure 6I, arrow). Afterward, PRL-3 signals

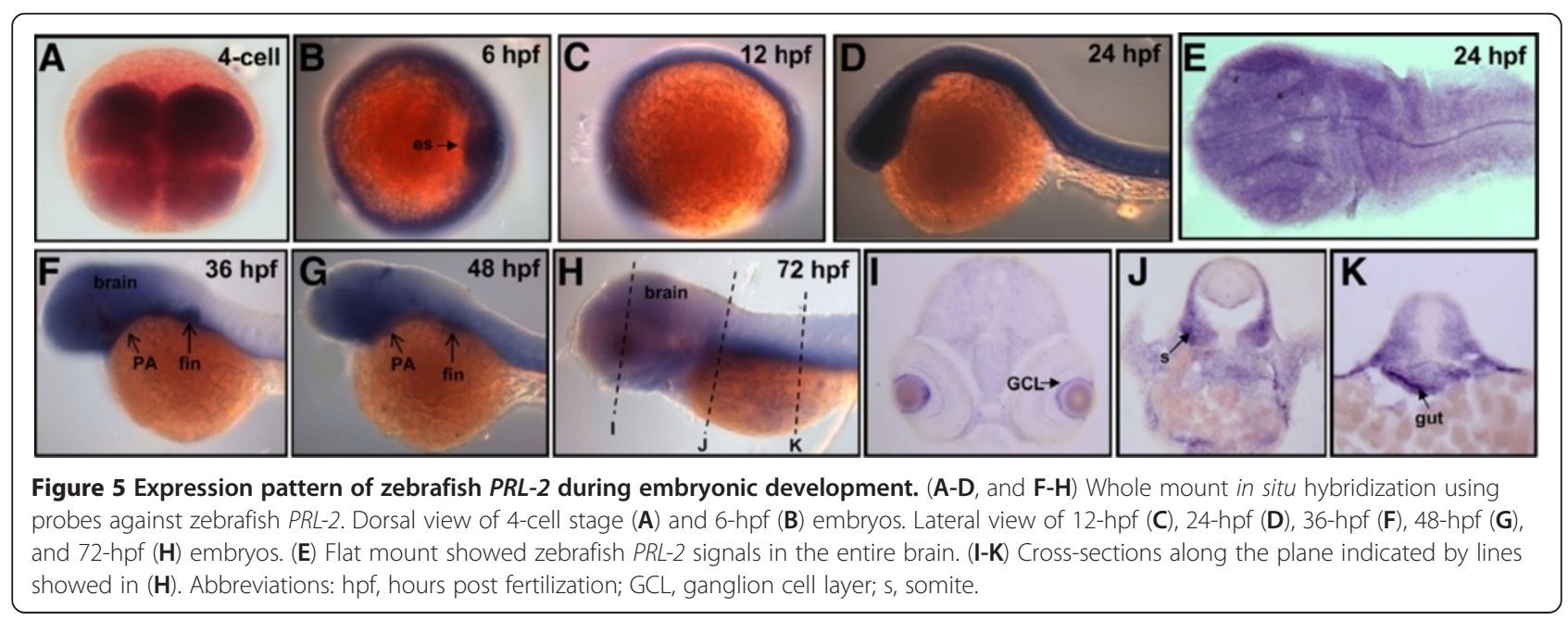




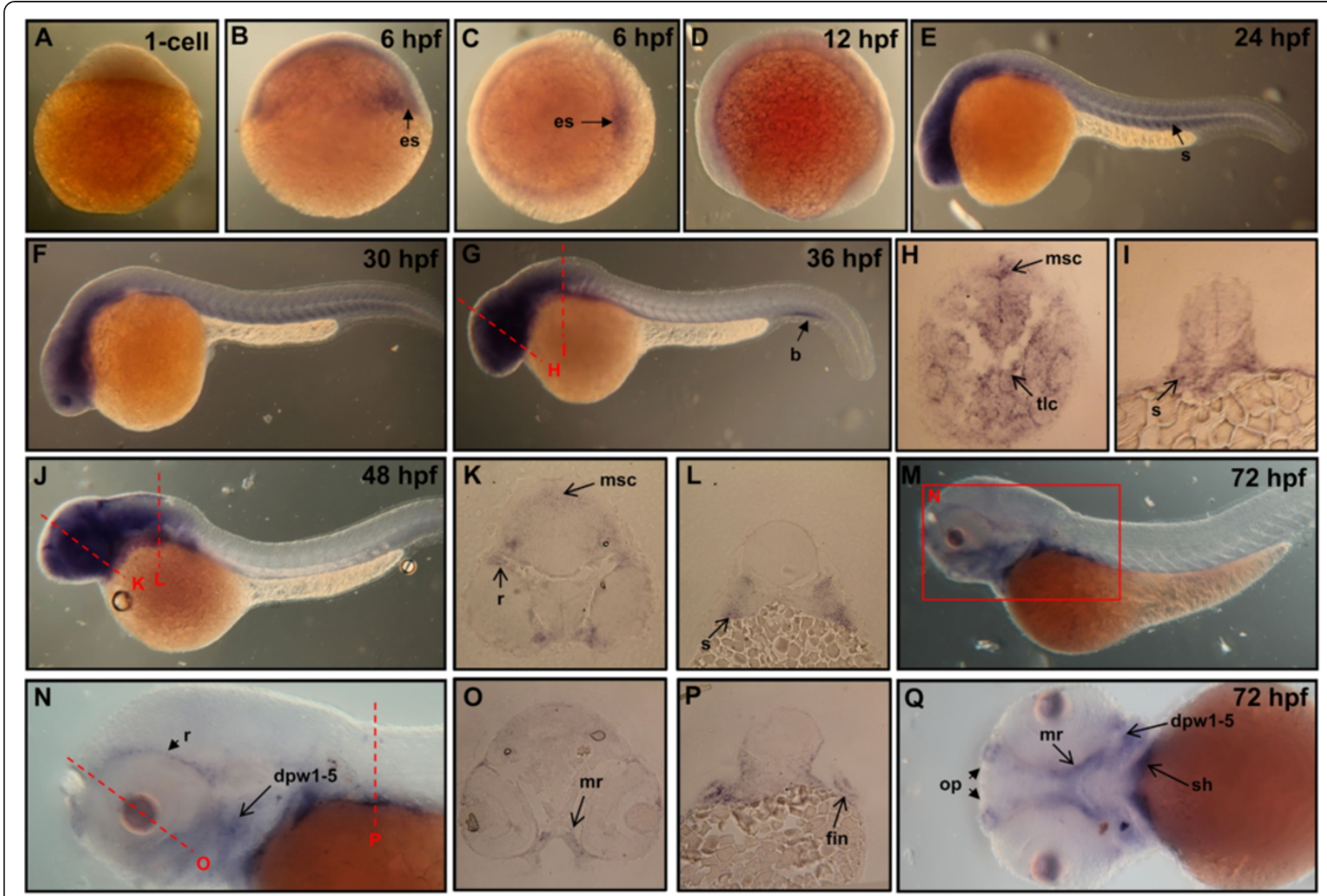

Figure 6 Expression pattern of zebrafish PRL-3 during embryonic development. (A-Q) Whole mount in situ hybridization using probes against zebrafish PRL-3. Dorsal view of a 6-hpf (C) embryo. Lateral view of 1-cell (A), 6-hpf (B), 12-hpf (D), 24-hpf (E), 30 hpf (F), 36-hpf (G), 48-hpf (J), and 72-hpf (M, N) embryos. (H-I, K-L and $\mathbf{O}-\mathbf{P})$ Cross-sections along the plane indicated by lines showed in $(\mathbf{G}, \mathbf{J}$, and $\mathbf{N})$, respectively. (Q) Ventral view of a 72-hpf embryo. Abbreviations: b, blood island; dpw1-5, dorsal pharyngeal wall 1-5; es, embryonic shield; mr, medial rectus; msc, mesencephalon; s, somite; sh, sternohyoideus; pa, pharyngeal arch; r, retina; op, olfactory placode; tlc, telencephalon.

at head region persisted while its expression in somite diminished (Figures 6G-H). It is interesting to note that zebrafish $P R L-3$ was transiently expressed at blood island (b) at $36 \mathrm{hpf}$ (Figure 6G, arrow), suggesting that $P R L-3$ might play a role on blood cell maturation. By 72-hpf, zebrafish $P R L-3$ signals were expressed at a small subset of head muscles including dorsal pharyngeal wall 1-5 (Figure 6Q, arrow), medial rectus (Figures $6 \mathrm{O}$ and $\mathrm{Q}$, arrows), sternohyoideus (Figure 6Q, arrow). In addition, zebrafish $P R L-3$ signals can be observed in retina (Figure 6N, arrow), fin (Figure 6P, arrow), and olfactory placode (Figure 6Q, arrow).

\section{Amphioxus $P R L$ is initially expressed throughout the embryo but later concentrated to the anterior neural tube in the larva}

To understand the evolution of $P R L$ developmental expression patterns, we analyzed $P R L$ gene expression in basal chordate amphioxus by whole mount in situ hybridization. At blastula stage, weak amphioxus $P R L$ expression was detected throughout the entire embryo
(Figure 7A), and this ubiquitous expression continued to gastrula stage (Figure 7B-D). At neurula stages, amphioxus $P R L$ transcripts were still detected throughout the embryo, but we noticed that the signals appeared to be stronger in the dorsal side of the embryos (Figure 7E-H, arrowheads). During the early larval stage, we found amphioxus PRL transcripts were more concentrated in the anterior neural tube at the cerebral vesicle of amphioxus (Figure 7I-J), although some weak signals were still detectable throughout the body.

\section{Discussion}

PRL is an evolutionary conserved protein family that is widely distributed in many species among the protostomes and deuterostomes. Through database search, it is evident that all of the examined vertebrate genomes possess three PRL genes, whereas invertebrate animals including C. elegans, Drosophila, Sea urchin, and the basal chordate amphioxus Branchiostoma floridae have only one $P R L$ gene. Our molecular phylogenetic analysis suggests that the three vertebrate $P R L$ genes may be the 

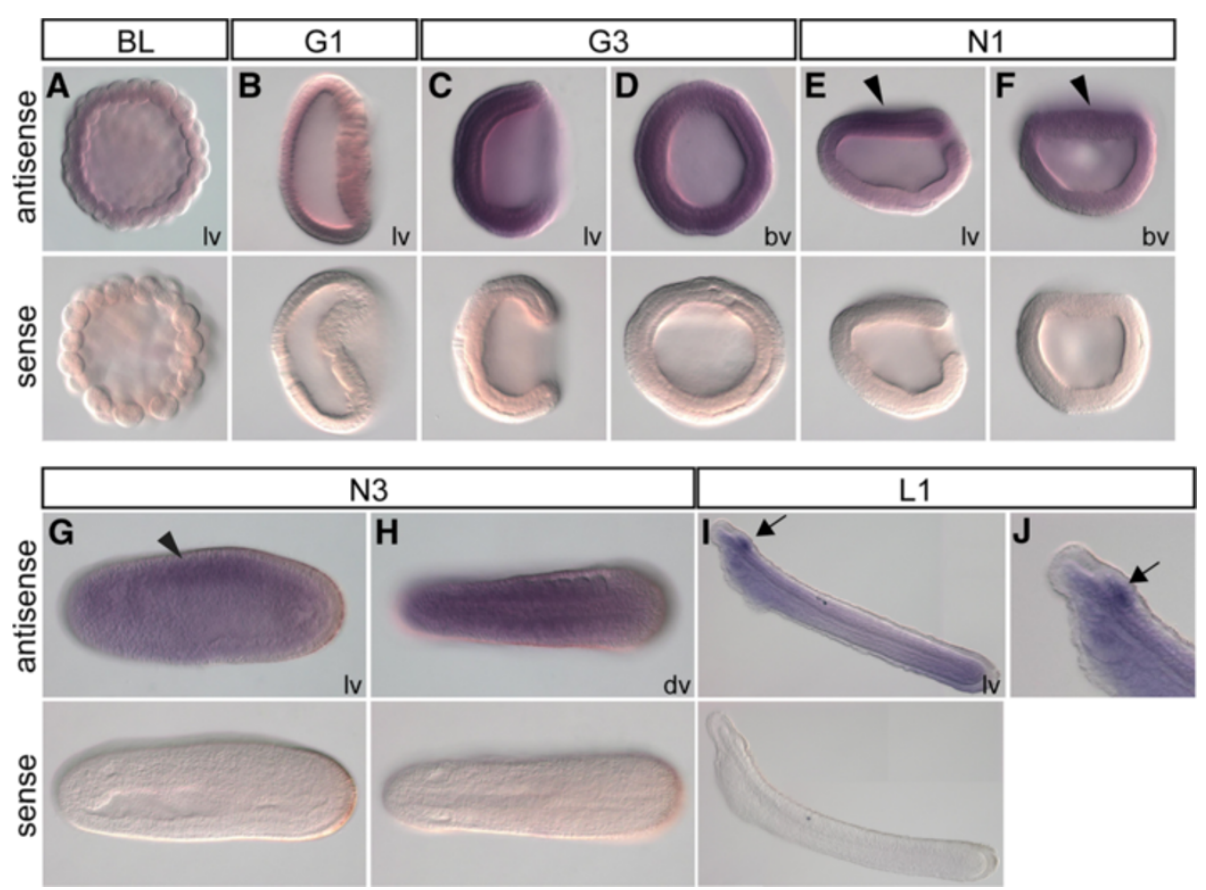

Figure 7 Expression pattern of amphioxus PRL during embryonic development. (A-I) Upper images show results of whole mount in situ hybridization using antisense probes against amphioxus $P R L$; lower images are whole mount in situ hybridization using sense amphioxus $P R L$ probes as negative controls. (A) Blastula stage, (B) early gastrula stage, (C-D) mid gastrula stage, (E-F) early neurula stage, (G-H) late neurula stage, (I) 36-hpf larval stage; IV, lateral view; bv, blastopore view; dv, dorsal view; arrowheads indicate weak expression in the dorsal part of the embryos; arrow in (I) indicates expression in the cerebral vesicle. (J) Enlarged view of the anterior end of the same 36-hpf larva in (I) showing amphioxus $P R L$ expression in the cerebral vesicle (arrowhead).

product of gene duplication events that happened at the base of the vertebrate lineage. By comparing wholegenome synteny patterns, it has been shown that the vertebrate genome had undergone two rounds of wholegenome duplication during early vertebrate evolution [29]. We analyzed the synteny patterns between amphioxus and human genomic regions around $P R L$ genes and found that the single amphioxus PRL gene is located on the Branchiostoma floridae genomic scaffold 233 (Additional file 3: Figure S3A), while the three human $P R L$ paralogues are located on three different chromosomes. We identified some conserved synteny patterns among amphioxus $P R L$ and human $P R L-1$ and $P R L-2$ (Additional file 3: Figure S3B); however, no synteny conservation could be identified around the genomic region harboring $P R L-3$ on human chromosome 8 , which may be due to genomic rearrangement after duplication. In fact, we also detected extensive genomic rearrangements that may have caused translocation (such as MDGA1 on human chromosome 6) and tandem duplication of genes (such as AGO1, AGO3 on AGO4 on human chromosome 1) around the vertebrate $P R L-1$ and $P R L-2$ genomic regions (Additional file 3: Figure $\mathrm{S} 3 \mathrm{~B}$ ), suggesting that after two rounds of whole-genome duplication, vertebrate genomes have experienced extensive rearrangements during evolution.

In mammals, some of the expression profiles of $P R L$ phosphatase have been characterized at either the protein and/or mRNA levels in normal tissues (Table 2). However, embryonic expression patterns of PRL orthologs have not yet been characterized in teleosts and invertebrates. In this study, we characterized all of the three zebrafish $P R L$ genes, Drosophilia $P R L$, as well as amphioxus $P R L$, and examined their embryonic expression patterns. In vertebrates including zebrafish, rat, mouse, and human, most of PRL- 1 and PRL- 2 are found to be expressed in the neuronal cell lineage (Table 2 and Figures 4 and 5). With regard to Drosophila PRL, it was detected strongly in the ventral nerve cord during embryogenesis (Figure 2), and the expression of amphioxus PRL gene is also detected in the anterior central nervous system (Figure 7). These results suggest that the biological role of "prototypic PRL" might be involved in the nervous system development.

In contrast to general neuronal expression of $P R L-1$, $P R L-2$, the expression of mammalian and zebrafish $P R L-3$ phosphatases are preferential in mesodermal cell lineage, such as heart, skeletal muscle, and pre-erythrocytes (Table 2 
Table 2 Expression profiles of PRL family genes in embryos, larvae, and adult normal tissues

\begin{tabular}{|c|c|c|c|}
\hline Species & Gene & Embryonic and/or larval expression domains & Expression in adult tissue \\
\hline \multirow[t]{3}{*}{ Rat } & PRL-1 & Brain [13] & $\begin{array}{l}\text { In brain, skeletal muscle, and digestive epithelial tissues } \\
{[1,13-15]}\end{array}$ \\
\hline & PRL-2 & ND & Ubiquitous expression [16] \\
\hline & PRL-3 & Fetal heart [17] & ND \\
\hline \multirow[t]{3}{*}{ Mouse } & PRL-1 & $\begin{array}{l}\text { Most tissues except heart and skeletal } \\
\text { muscle [18] }\end{array}$ & ND \\
\hline & PRL-2 & ND & Preferential in skeletal muscle [3] \\
\hline & PRL-3 & ND & $\begin{array}{l}\text { Preferential in heart and skeletal muscle; also in small } \\
\text { intestine }[3,21]\end{array}$ \\
\hline \multirow[t]{3}{*}{ Human } & PRL-1 & ND & Ubiquitous expression except brain cortex [19] \\
\hline & PRL-2 & ND & Ubiquitous expression [19] \\
\hline & PRL-3 & ND & $\begin{array}{l}\text { Mainly in heart and skeletal muscle; pre-erythrocytes } \\
\text { in bone marrow }[17,20]\end{array}$ \\
\hline \multirow[t]{3}{*}{ Zebrafish } & PRL-1 & Mainly in neuronal cell lineage & ND \\
\hline & PRL-2 & Mainly in neuronal cell lineage & ND \\
\hline & PRL-3 & Mainly in skeletal muscle & ND \\
\hline Amphioxus & $P R L$ & Ubiquitous in embryos; Central nervous system in larvae & ND \\
\hline Drosophila & $P R L$ & $\begin{array}{l}\text { Developing mid-gut and central nervous system in } \\
\text { embryos; Imaginal discs in larvae }\end{array}$ & ND \\
\hline
\end{tabular}

Abbreviation: ND, Not determined.

and Figure 6). Thus, we propose that the ancestral $P R L$ is predominantly expressed in neuronal cell lineage, especially in the central nervous system. In the vertebrate lineage, two of the duplicated $P R L$ paralogues, $P R L-1$ and $P R L-2$, retained this neuronal expression, while the third paralogue, $P R L-3$, may have evolved new function and obtained more specific expression in the mesodermal cell lineage.

\section{Conclusions}

In summary, our study characterizes the distinct expression patterns of three structurally related zebrafish $P R L$ genes and those of Drosophila and amphioxus $P R L$ homologues. Our comparisons provide interesting insight into the evolution of PRL genes and their embryonic expression patterns, and highlight the possible consequence of gene duplication events on the neofunctionalization of duplicated genes during vertebrate evolution.

\section{Methods}

\section{Antibody generation}

To generate a rabbit anti-PRL antibody, we subcloned the full length dmPRL CDS from cDNA clone RE40268 into pET-32a vector for producing recombinant His-tagged dmPRL proteins in E.coli. The dmPRL recombinant protein purification together with the generation of the antidmPRL polyclonal rabbit antiserum were performed by GeneTex International Corp. approved by the Industrial Development \& Investment Promotion Committee of Hsin-Chu City, Taiwan.

\section{Drosophila whole mount immunostaining}

For embryo immunostaining, collected embryos were washed with $0.4 \% \mathrm{NaCl}, 0.1 \%$ Triton $\mathrm{X}-100$, dechorionated with $100 \%$ bleach for $2 \mathrm{~min}$, and washed with deionized water. Embryos were fixed for $20 \mathrm{~min}$ in $4 \%$ formaldehyde with heptane. After removal of the fixative, embryos were washed with methanol several times and rehydrated into phosphate buffered Tris. Embryos were blocked with $2 \%$ bovine serum albumin in PBT (PBS containing $0.2 \%$ Tween-20) for 1 hour, and incubated overnight at $4^{\circ} \mathrm{C}$ in primary antibody diluted in PBS. The embryos were then washed 3 times for $20 \mathrm{~min}$ each in PBT, and then incubated for $2 \mathrm{~h}$ at room temperature in secondary antibody in PBT. Following three 30 min washes in PBT, the embryos were mounted in anti-fade mounting solution (PBS containing 50\% glycerol and 2\% DABCO). For eye disc immunostaining, hand dissected eye discs were fixed for $20 \mathrm{~min}$ in $4 \%$ formaldehyde. After removal of the fixative, embryos were washed several times in PBST (PBS containing 0.3\% Triton X-100). Eye discs were blocked with $2 \%$ bovine serum albumin in PBST for 1 hour, and incubated overnight at $4^{\circ} \mathrm{C}$ in primary antibody diluted in PBS. The eye discs were then washed 3 times for $20 \mathrm{~min}$ each in PBST, and then incubated for $2 \mathrm{~h}$ at room temperature in secondary antibody in PBST. Following three $30 \mathrm{~min}$ washes in PBT, the discs were mounted in anti-fade mounting solution. The following primary antibodies were used: rabbit anti-PRL, rat anti-ELAV (Developmental Studies Hybridoma Bank), mouse anti-HRP (Jackson 
Labs). Fluorescent-labeled secondary antibodies used goat-anti-rabbit Alexa Fluor 488 (Invitrogen), goat antirat Alexa Fluor 633 (Invitrogen), and goat anti-mouse Alexa Fluor 633 (Invitrogen). F-actin was labeled by Alexa Flour 633 conjugated phalloidin (Invitrogen).

\section{Drosophila whole mount in situ hybridization}

The full length CDS of $d m P R L$ cDNA were cloned into pGEM-3Z vector and used to synthesize digoxigenin (DIG) labeled RNA probes for in situ hybridization. Template preparation, probe synthesis, and procedure for whole mount in situ hybridization were performed as previously described [30]. All embryos were observed under a Nikon Eclipse E800 microscope equipped with Nomarski differential interference contrast optics and a CCD camera.

\section{Zebrafish strain and embryo staging}

Mature zebrafish (AB strain) were raised at the zebrafish facility of the Life Sciences Development Center, Tamkang University. All animal experiments in this study were approved by Tamkang University and performed in accordance with the "Animal Research: Reporting in vivo Experiments" guideline issued by regional animal ethic committee. The fish were maintained at $28^{\circ} \mathrm{C}$ with a photoperiod of $14 \mathrm{~h}$ light and $10 \mathrm{~h}$ dark, in an aquarium supplied with freshwater and aeration [31]. Embryos were produced using standard procedures and were staged according to standard criteria [32].

\section{Zebrafish whole mount in situ hybridization, cryosection and imaging}

The procedures for whole mount in situ hybridization, and cryosection have been described previously [33], except that the full length of individual zebrafish $P R L-1$, $P R L-2$, and $P R L-3$ coding sequences were used to produce digoxigenin-labeled probes. All embryos were observed under a Leica DM 2500 microscope equipped with Nomarski differential interference contrast optics (Kramer Scientific) and a digital camera (Cannon, Japan).

\section{Identification of amphioxus PRL homologue and whole mount in situ hybridization}

Amphioxus homologue of $P R L$ gene was identified from Branchiostoma floridae draft genome [29] by BLAST search using Drosophila PRL protein as queries. Identified Gene models were subsequently used to search the amphioxus EST database and cDNA collection (B. floridae Gene Collection Release 1 [34]) to isolate the corresponding cDNA clones. The identified cDNA clones were sequenced from both ends by M13 forward and reverse primers as well as internal primers to obtain the complete nucleotide sequence of the inserts. At the end, we identified three different cDNA isoforms (amphioxus cDNA ID: bfad016c08, bfad039b03, and bfad043g05) representing one single $P R L$ gene in amphioxus (see Results).

Gravid animals of the amphioxus (B. floridae) were collected in Tampa Bay, Florida USA, during the summer breeding season. Gametes were obtained by electric stimulation. Fertilization and subsequent culturing of the embryos were carried out as previously described [35]. Amphioxus PRL cDNA clones isolated from the aforementioned cDNA collection were used to synthesize digoxigenin (DIG) labeled anti-sense and sense RNA probes for in situ hybridization. Template preparation, probe synthesis, and procedure for single-color in situ hybridization were performed as previously described [36]. Whole-mount in situ hybridization on amphioxus embryos was performed as previously described [37]. Images of embryos were taken using a Zeiss Axio Imager A1 microscope with a Zeiss AxioCam MRc CCD camera. Probes generated from the three amphioxus PRL cDNA isoforms gave us identical pattern, thus we only present results from one cDNA clone, bfad016c08, which gave us the strongest signals.

\section{Additional files}

\begin{abstract}
Additional file 1: Figure S1. Genomic structure of amphioxus PRL gene. The thick black line represents the $33 \mathrm{~kb}$ genomic DNA region on scaffold Bf_V2-223 that contains the PRL locus. Grey boxes above the genomic scaffold are the locations of the predicted exons. Patterns of exon usage for each cDNA isoforms are depicted with colored boxes under the genomic scaffold. Red lines indicate the position of start and stop codon. Scale bar: $1 \mathrm{~kb}$.
\end{abstract}

Additional file 2: Figure S2. Anti-dmPRL rabbit antiserum recognized both endogenous dmPRL and GFP-tagged dmPRL in ovary and embryo lysates. (A) Anti-dmPRL rabbit antiserum recognized both the GFP-tagged dmPRL fusion protein (lane 1, arrow) and endogenous dmPRL (lane 1-3, arrowheads) in both ovary and embryo lysates. (B) The same PVDF membrane of (A) was stripped and re-probed with anti-GFP antibody to show the GFP-dmPRL fusion protein of GFP-dmPRL expressing ovary lysate. (C) The same PVDF membrane of $(\mathbf{A})$ was stripped and re-probed with anti-Tubulin antibody as a loading control. Asterisk indicates the non-specific band recognized by anti-dmPRL rabbit antiserum. Lane 1: GFP-dmPRL expressing ovary lysate. Lane 2: wild-type ovary lysate. Lane 3: wild-type embryo lysate.

Additional file 3: Figure S3. Synteny comparison between amphioxus and human PRL chromosomal regions. (A) The single amphioxus PRL gene is located on Scaffold 233 in Version 2 assembly (scaffold_104 of Version 1 assembly in the JGl genome browser). Arrows represent the amphioxus PRL and its neighboring genes on the scaffold in the direction of transcription. The numbers represent the distance (in kilobase) between neighboring genes. In this schematic drawing the distances between genes are not in scale. (B) The three human PRL paralogues (PRL-1, PRL-2, and PRL-3) are located on three different chromosones. Black arrows highlight the positions of $P R L$ paralogues and the traces of conserved synteny between amphioxus and human PRL chromosome region. Human synteny information was retrieved from NCBI Map Viewer database at http://www.ncbi.nlm.nih.gov/mapview/

\section{Abbreviations}

CDS: Coding sequence; CNS: Central nervous system; DIG: Digoxigenin; hpf: Hours post fertilization; PRL: Phosphatase of regenerating liver; VNC: Ventral nerve cord. 


\section{Competing interests}

The authors declare that they have no competing interests.

\section{Authors' contributions}

$M D L, J K Y$, and YHC conceived and designed the experiments. HTL, SCW, $H R L, H L H, K W C, Y D C$, and MLH performed the experiments. MDL, JKY, and YHC wrote the paper. All authors read and approved the final manuscript.

\section{Acknowledgements}

We are grateful to the Fly Core in Taiwan for fly stocks and reagents. We thank the technical supports provided by sequencing core facility of the National Yang-Ming University Genome Research Center (YMGC). The Sequencing Core Facility is supported by National Research Program for Genomic Medicine (NRPGM), National Science Council. The confocal microscopy was provided by the office of research and development at Tzu-Chi University. This work is supported by the National Sciences Council (NSC) of Taiwan (NSC101-2311-B-320-001-MY3) and Tzu Chi Foundation (610300239) to Ming-Der Lin. NSC99-2627-B-001-003, NSC101-2923-B-001004-MY2, and the Career Development Award (AS-98-CDA-L06) from Academia Sinica, Taiwan, to Jr-Kai Yu. NSC101-2313-B-032-001-MY3 to Yau-Hung Chen.

\section{Author details}

'Department of Molecular Biology and Human Genetics, Tzu-Chi University, No.701, Zhongyang Rd., Sec 3, Hualien 97004, Taiwan. ${ }^{2}$ Department of Life Sciences, Tzu-Chi University, No.701, Zhongyang Rd, Sec 3, Hualien 97004, Taiwan. ${ }^{3}$ Department of Life Science, National Chung-Cheng University, No.168, Sec. 1, University Rd, Min-Hsiung Township, Chia-yi County 621, Taiwan. ${ }^{4}$ Institute of Cellular and Organismic Biology, Academia Sinica, 128 Academia Road, Sec. 2, Nankang, Taipei 11529, Taiwan. ${ }^{5}$ Department of Chemistry, Tamkang University, 151 Ying-Chuan Rd., Tamsui, New Taipei City 25137, Taiwan

Received: 25 January 2013 Accepted: 29 April 2013

Published: 4 May 2013

\section{References}

1. Diamond RH, Cressman DE, Laz TM, Abrams CS, Taub R: PRL-1, a unique nuclear protein tyrosine phosphatase, affects cell growth. Mol Cell Biol 1994, 14:3752-3762.

2. Mohn KL, Laz TM, Hsu JC, Melby AE, Bravo R, Taub R: The immediate-early growth response in regenerating liver and insulin-stimulated $\mathrm{H}-35$ cells: comparison with serum-stimulated $3 \mathrm{~T} 3$ cells and identification of 41 novel immediate-early genes. Mol Cell Biol 1991, 11:381-390.

3. Zeng Q, Hong W, Tan YH: Mouse PRL-2 and PRL-3, two potentially prenylated protein tyrosine phosphatases homologous to PRL-1. Biochem Biophys Res Commun 1998, 244:421-427.

4. Bessette DC, Qiu D, Pallen CJ: PRL PTPs: mediators and markers of cancer progression. Cancer Metastasis Rev 2008, 27:231-252.

5. Kato H, Semba S, Miskad UA, Seo Y, Kasuga M, Yokozaki H: High expression of PRL-3 promotes cancer cell motility and liver metastasis in human colorectal cancer: a predictive molecular marker of metachronous liver and lung metastases. Clin Cancer Res 2004, 10:7318-7328.

6. Li J, Guo K, Koh WW, Tang JP, Gan BQ, Shi H, Li HX, Zeng Q: Generation of PRL-3- and PRL-1-specific monoclonal antibodies as potential diagnostic markers for cancer metastases. Clin Cancer Res 2005, 11:2195-2204.

7. Han H, Bearss DJ, Browne LW, Calaluce R, Nagle RB, Von Hoff DD: Identification of differentially expressed genes in pancreatic cancer cells using CDNA microarray. Cancer Res 2002, 62:2890-2896.

8. Wang J, Kirby CE, Herbst R: The tyrosine phosphatase PRL-1 localizes to the endoplasmic reticulum and the mitotic spindle and is required for normal mitosis. J Biol Chem 2002, 277:46659-46668.

9. Wang Q, Holmes DI, Powell SM, Lu QL, Waxman J: Analysis of stromal-epithelial interactions in prostate cancer identifies PTPCAAX2 as a potential oncogene. Cancer Lett 2002, 175:63-69.

10. Hardy S, Wong NN, Muller WJ, Park M, Tremblay ML: Overexpression of the protein tyrosine phosphatase PRL-2 correlates with breast tumor formation and progression. Cancer Res 2010, 70:8959-8967.

11. Radke I, Gotte M, Kersting C, Mattsson B, Kiesel L, Wulfing P: Expression and prognostic impact of the protein tyrosine phosphatases PRL-1, PRL-2, and PRL-3 in breast cancer. Br J Cancer 2006, 95:347-354.
12. Stephens BJ, Han H, Gokhale V, Von Hoff DD: PRL phosphatases as potential molecular targets in cancer. Mol Cancer Ther 2005, 4:1653-1661.

13. Takano S, Fukuyama H, Fukumoto M, Kimura J, Xue JH, Ohashi H, Fujita J: PRL-1, a protein tyrosine phosphatase, is expressed in neurons and oligodendrocytes in the brain and induced in the cerebral cortex following transient forebrain ischemia. Brain Res Mol Brain Res 1996, 40:105-115.

14. Diamond RH, Peters C, Jung SP, Greenbaum LE, Haber BA, Silberg DG, Traber PG, Taub R: Expression of PRL-1 nuclear PTPase is associated with proliferation in liver but with differentiation in intestine. Am J Physiol 1996, 271:G121-G129.

15. Kong W, Swain GP, Li S, Diamond RH: PRL-1 PTPase expression is developmentally regulated with tissue-specific patterns in epithelial tissues. Am J Physiol Gastrointest Liver Physiol 2000, 279:G613-G621.

16. Carter DA: Expression of a novel rat protein tyrosine phosphatase gene. Biochim Biophys Acta 1998, 1442:405-408.

17. Guo K, Li J, Wang H, Osato M, Tang JP, Quah SY, Gan BQ, Zeng Q: PRL-3 initiates tumor angiogenesis by recruiting endothelial cells in vitro and in vivo. Cancer Res 2006, 66:9625-9635.

18. Rundle $\mathrm{CH}$, Kappen C: Developmental expression of the murine Prl-1 protein tyrosine phosphatase gene. J Exp Zool 1999, 283:612-617.

19. Zeng Q, Si X, Horstmann H, Xu Y, Hong W, Pallen CJ: Prenylation-dependent association of protein-tyrosine phosphatases PRL-1, -2 , and -3 with the plasma membrane and the early endosome. J Biol Chem 2000, 275:21444-21452.

20. Dumaual CM, Sandusky GE, Crowell PL, Randall SK: Cellular localization of PRL-1 and PRL-2 gene expression in normal adult human tissues. $J$ Histochem Cytochem 2006, 54:1401-1412.

21. Matter WF, Estridge T, Zhang C, Belagaje R, Stancato L, Dixon J, Johnson B, Bloem L, Pickard T, Donaghue M, et al: Role of PRL-3, a human musclespecific tyrosine phosphatase, in angiotensin-II signaling. Biochem Biophys Res Commun 2001, 283:1061-1068.

22. Saitou N, Nei M: The neighbor-joining method: a new method for reconstructing phylogenetic trees. Mol Biol Evol 1987, 4:406-425.

23. Tamura K, Peterson D, Peterson N, Stecher G, Nei M, Kumar S: MEGA5: molecular evolutionary genetics analysis using maximum likelihood, evolutionary distance, and maximum parsimony methods. Mol Biol Evol 2011, 28:2731-2739.

24. Zhang ZY: Protein tyrosine phosphatases: structure and function, substrate specificity, and inhibitor development. Annu Rev Pharmacol Toxicol 2002, 42:209-234.

25. Zhang ZY: Protein-tyrosine phosphatases: biological function, structural characteristics, and mechanism of catalysis. Crit Rev Biochem Mol Biol 1998, 33:1-52.

26. Cates CA, Michael RL, Stayrook KR, Harvey KA, Burke YD, Randall SK, Crowell $\mathrm{PL}$, Crowell DN: Prenylation of oncogenic human PTP(CAAX) protein tyrosine phosphatases. Cancer Lett 1996, 110:49-55.

27. Sun JP, Luo Y, Yu X, Wang WQ, Zhou B, Liang F, Zhang ZY: Phosphatase activity, trimerization, and the $\mathrm{C}$-terminal polybasic region are all required for PRL1-mediated cell growth and migration. J Biol Chem 2007, 282:29043-29051.

28. Si X, Zeng Q, Ng CH, Hong W, Pallen CJ: Interaction of farnesylated PRL-2, a protein-tyrosine phosphatase, with the beta-subunit of geranylgeranyltransferase II. J Biol Chem 2001, 276:32875-32882.

29. Putnam NH, Butts T, Ferrier DE, Furlong RF, Hellsten U, Kawashima T, Robinson-Rechavi M, Shoguchi E, Terry A, Yu JK, et al: The amphioxus genome and the evolution of the chordate karyotype. Nature 2008, 453:1064-1071.

30. Legendre F, Cody N, lampietro C, Bergalet J, Lefebvre FA, Moquin-Beaudry G, Zhang O, Wang X, Lecuyer E: Whole mount RNA fluorescent in situ hybridization of drosophila embryos. J Vis Exp 2013, 71:e50057. doi:10.3791/50057.

31. Chen $Y H$, Lin $Y T$, Lee $G H$ : Novel and unexpected functions of zebrafish CCAAT box binding transcription factor (NF-Y) B subunit during cartilages development. Bone 2009, 44:777-784.

32. Kimmel CB, Ballard WW, Kimmel SR, Ullmann B, Schilling TF: Stages of embryonic development of the zebrafish. Dev Dyn 1995, 203:253-310.

33. Peng $\mathrm{HC}$, Wang $\mathrm{YH}$, Wen $\mathrm{CC}$, Wang $\mathrm{WH}$, Cheng $\mathrm{CC}$, Chen $\mathrm{YH}$ : Nephrotoxicity assessments of acetaminophen during zebrafish embryogenesis. Comp Biochem Physiol C Toxicol Pharmacol 2010, 151:480-486. 
34. Yu JK, Wang MC, Shin I, Kohara Y, Holland LZ, Satoh N, Satou Y: A cDNA resource for the cephalochordate amphioxus Branchiostoma floridae. Dev Genes Evol 2008, 218:723-727.

35. Holland LZ, Yu JK: Cephalochordate (amphioxus) embryos: procurement, culture, and basic methods. Methods Cell Biol 2004, 74:195-215.

36. Wu HR, Chen YT, Su YH, Luo YJ, Holland LZ, Yu JK: Asymmetric localization of germline markers vasa and nanos during early development in the amphioxus Branchiostoma floridae. Dev Biol 2011, 353:147-159.

37. Lu TM, Luo YJ, Yu JK: BMP and delta/notch signaling control the development of amphioxus epidermal sensory neurons: insights into the evolution of the peripheral sensory system. Development 2012, 139:2020-2030.

doi:10.1186/1471-213X-13-18

Cite this article as: Lin et al:: Expression of phosphatase of regenerating liver family genes during embryogenesis: an evolutionary developmental analysis among Drosophila, amphioxus, and zebrafish. BMC Developmental Biology 2013 13:18.

\section{Submit your next manuscript to BioMed Central and take full advantage of:}

- Convenient online submission

- Thorough peer review

- No space constraints or color figure charges

- Immediate publication on acceptance

- Inclusion in PubMed, CAS, Scopus and Google Scholar

- Research which is freely available for redistribution 